\title{
AN OPTIMIZED LOW-CHARGE CONFIGURATION OF THE LINAC COHERENT LIGHT SOURCE *
}

\author{
P. Emma ${ }^{\dagger}$, Z. Huang, C. Limborg-Deprey, J. Wu, (SLAC) \\ W. Fawley, M. Zolotorev, (LBNL), S. Reiche, (UCLA)
}

\begin{abstract}
The Linac Coherent Light Source (LCLS) is an x-ray free-electron laser (FEL) project based on the SLAC linac. The nominal parameter set is founded on a 1-nC bunch charge and normalized emittance of about 1 micron. Many of the most challenging issues are associated with the relatively high charge. The study described here uses a $0.2-\mathrm{nC}$ bunch charge and $0.85-\mu \mathrm{m}$ emittance with only $30 \mathrm{~A}$ of peak current in the injector, producing the same FEL saturation length. The resulting performance is more stable, has negligible resistive wakefield, greatly reduced CSR effects, and no transverse wakefield emittance dilution in the linac, with no change to the baseline engineering design.
\end{abstract}

\section{INTRODUCTION}

The LCLS is a SASE X-ray FEL project at SLAC [1], just beginning construction and scheduled for first FEL light in 2009. The nominal operating point uses a 1$\mathrm{nC}$ bunch charge, which is typical in other FEL projects (see, e.g., Ref. [2]). Many of the most challenging issues, such as emittance generation, wakefields, and coherent synchrotron radiation (CSR), are associated with the high bunch charge. In the LCLS in particular, with its strong linac wakefields, the bunch compression process produces sharp temporal horns at the head and tail of the bunch with degraded local emittance, effectively wasting much of the charge. The sharp horns intensify CSR in the bends and further drive a strong resistive-wall wakefield in the long FEL undulator. Although these issues are not insurmountable, they suggest a lower charge may be more suitable.

In addition to these accelerator physics issues associated with the high bunch charge, the normalized transverse emittance of $1 \mu \mathrm{m}$ assumed for the nominal 1-nC configuration is not ideally matched to the transverse phase space properties of the FEL radiation. In fact, the transverse phase space area of such an electron beam at the undulator (at $14 \mathrm{GeV}$ ) is about three times as large as the phase space area of the coherent radiation at $1.5 \AA$. Decreasing the bunch charge should also reduce the transverse emittance generated in the RF gun, which will allow for a better match with the radiation emittance and hence a more efficient FEL interaction in the undulator. Since the quantitative scaling of the transverse emittance with the bunch charge has not been demonstrated experimentally in the interesting parameter regime, we will make a conservative

\footnotetext{
* Work supported by US Department of Energy contract DE-AC0276 SF00515.

†Emma@SLAC.Stanford.edu
}

estimation about the reduction of the transverse emittance with the bunch charge in this paper and leave a possible emittance-matched configuration for a future study.

\section{PARAMETER CHOICES}

The bunch charge chosen here is based on practical issues. A level of $0.2 \mathrm{nC}$ is a substantial reduction of the bunch charge with respect to $1 \mathrm{nC}$, but is also within present diagnostics capabilities of the SLAC linac. We choose to maintain the present design parameters for the LCLS undulator in order to preserve the engineering baseline design and also keep the 1-nC option open.

Figure 1 shows a plot of the estimated saturation length, based on Xie's fitting formula [3], as a function of transverse normalized emittance, for several values of peak current, where the energy, energy spread, FEL wavelength, mean beta, undulator parameter, and undulator period are all held constant at $E=13.6 \mathrm{GeV}, \sigma_{\delta}=0.01 \%, \lambda_{r}=1.5$ $\AA,\langle\beta\rangle=30 \mathrm{~m}, K=3.5$, and $\lambda_{u}=3 \mathrm{~cm}$, respectively.

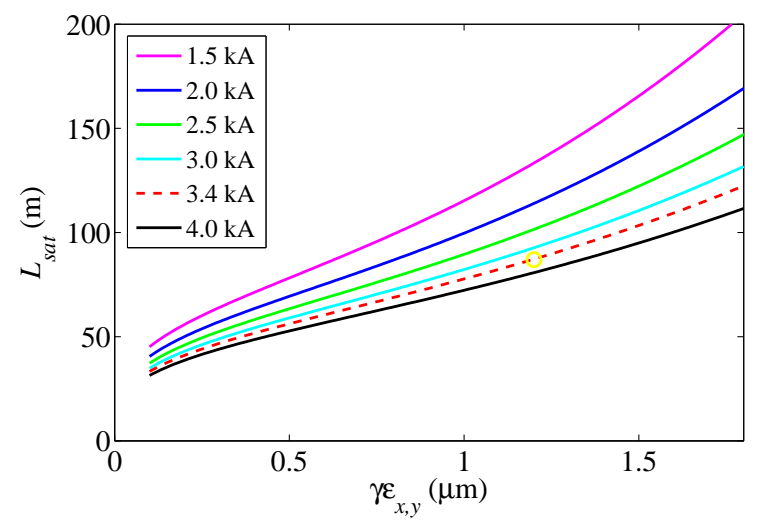

Figure 1: SASE saturation length [3] at $1.5 \AA$ as a function of transverse emittance for several values of peak current.

Allowing for a reasonable, but smaller emittance in the RF photo-cathode gun with 5-times less charge [4], and maintaining the SASE saturation length at about $87 \mathrm{~m}$, we select a $0.85-\mu \mathrm{m}$ emittance at a $2.1-\mathrm{kA}$ peak current, as opposed to the $1.2-\mu \mathrm{m}$ emittance at $3.4 \mathrm{kA}$ in the $1-\mathrm{nC}$ design.

The 1-nC design requires a $1.0-\mu \mathrm{m}$ time-sliced emittance from the RF gun, with a $1.2-\mu \mathrm{m}$ value in the FEL undulator, allowing for a $20 \%$ growth in the linac. In fact, detailed tracking studies, including CSR, wakefields, and various system errors, even at $1 \mathrm{nC}$, show the slice emittance increased only by a few percent over the linac. Therefore, we reduce the slice emittance growth tolerance to 
$\Delta \gamma \epsilon_{s} \approx 0.05 \mu \mathrm{m}$, and set a $0.8-\mu \mathrm{m}$ slice emittance goal in the RF gun, only $20 \%$ less than the $1-n C$ goal.

To produce a 2.1-kA peak current in the FEL with just $0.2-n C$ of charge requires a significantly shorter bunch length $(8 \mu \mathrm{m} \mathrm{rms})$ than the $22-\mu \mathrm{m}$ length required at $1 \mathrm{nC}$. The pulse-to-pulse jitter of the final peak current scales approximately with the total bunch compression factor [5], so we also use a shorter initial bunch length from the gun to limit the increase of the total compression factor (up from 40 to 70$)$. This requires a $560-\mu \mathrm{m}$ rms initial bunch length (6.5 ps FWHM), rather than an $840-\mu$ m length (10 ps). This 1.5-times shorter initial bunch length, and 5-times less charge means the peak current in the RF gun is only $30 \mathrm{~A}$, rather than $100 \mathrm{~A}$, which also makes a $20 \%$ emittance reduction in the gun a more reasonable expectation. Tracking results through the gun and injector using Parmela [6] are shown in Fig. 2 and Table 1 lists the two parameters sets.
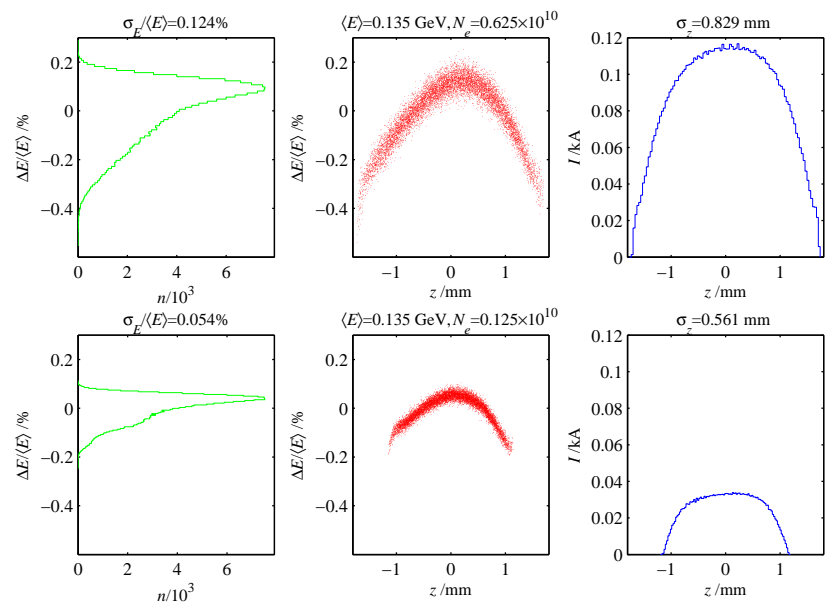

Figure 2: Longitudinal phase space at injector (135 MeV) with 1-nC (top) and 0.2-nC (bottom). Plots show energy profile (left), phase space (center), and longitudinal distribution (right). Bunch head at $z<0$.

Table 1: Parameters for 1-nC and 0.2-nC bunch charge.

\begin{tabular}{|l|c|c|c|c|}
\hline parameter & sym. & 1-nC & 0.2-nC & unit \\
\hline init. rms bunch lng. & $\sigma_{z_{0}}$ & 840 & 560 & $\mu \mathrm{m}$ \\
init. peak current & $I_{p k_{0}}$ & 100 & 30 & $\mathrm{~A}$ \\
init. slice emittance & $\gamma \epsilon_{0}$ & 1.0 & 0.80 & $\mu \mathrm{m}$ \\
final rms bunch lng. & $\sigma_{z_{f}}$ & 22 & 8.0 & $\mu \mathrm{m}$ \\
compression factor & $C$ & 40 & 70 & \\
final peak current & $I_{p k_{f}}$ & 3.4 & 2.1 & $\mathrm{kA}$ \\
final slice emittance & $\gamma \epsilon_{f}$ & 1.2 & 0.85 & $\mu \mathrm{m}$ \\
final rms $E$ spread & $\sigma_{\delta}$ & 1.0 & 1.0 & $10^{-4}$ \\
FEL satur. length & $L_{s a t}$ & 87 & 88 & $\mathrm{~m}$ \\
\hline
\end{tabular}

\section{LINAC TRACKING}

The linac RF and chicane compressor parameter changes required to produce $2.1-\mathrm{kA}$ beam are simple to apply and can be accomplished as simple operational changes, directed entirely from the control room. The RF phase changes are a few degrees and the chicane bend-field settings are only $5-10 \%$. In fact the second chicane (BC2) is $10 \%$ weaker, which further limits CSR effects.

Particle tracking through the linac using LiTrack [7] in $2 \mathrm{D}$ is shown in Fig. 3 for both the $1-\mathrm{nC}$ and $0.2-\mathrm{nC}$ cases. The advantage of the low-charge case is seen in comparison to the large peak current spikes that show up at $1 \mathrm{nC}$. More detailed 6D tracking using elegant [8] shows that transverse wakefields and dispersion errors due to BPM, quadrupole, and RF-structure misalignments are essentially eliminated at $0.2 \mathrm{nC}$ (see Fig. 4), due to both the lower charge and also the shorter average bunch length and its associated smaller chirped energy spread in misaligned magnets.
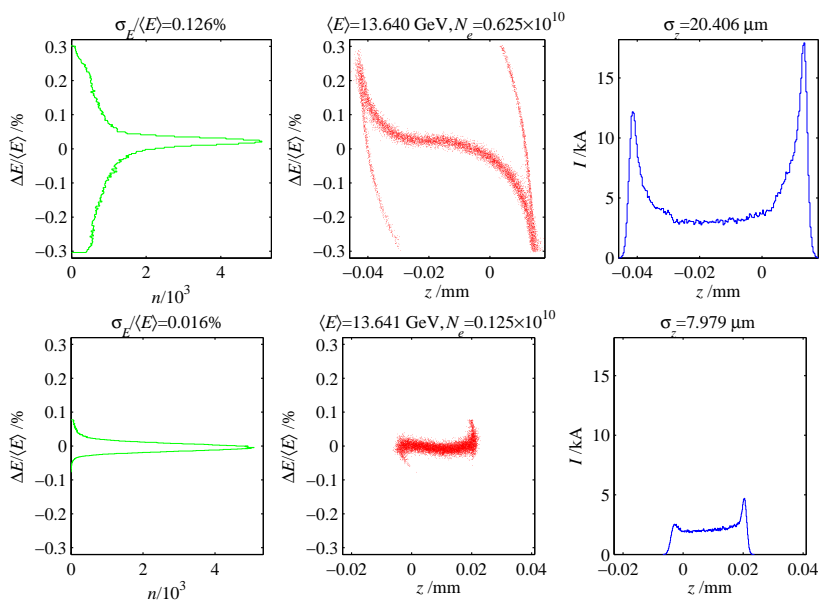

Figure 3: Longitudinal phase space in FEL at $13.6 \mathrm{GeV}$ with 1-nC (top) and 0.2-nC (bottom). Plots show the energy profile (left), phase space (center), and longitudinal distribution (right). Bunch head at $z<0$.

In addition, the micro-bunching instability induced by longitudinal space charge and CSR in the linac and chicanes, has a gain which is 3-times smaller for $0.2 \mathrm{nC}$ than for $1 \mathrm{nC}$ [9]. The laser-based beam heater used to Landau damp the instability also requires less power and is set to maintain the rms energy spread in the FEL undulator at $0.01 \%$. Finally, the relative horizontal projected emittance growth due to CSR in the $\mathrm{BC} 2$ chicane is reduced by three.

\section{UNDULATOR RESISTIVE WAKEFIELDS}

The resistive-wall wakefield in the FEL undulator has been calculated including the frequency dependence of the beam-pipe conductivity [10]. The sharp peak-current spikes at head and tail shown in the 1-nC case in Fig. 3 drive this resistive wake harder than does a Gaussian or rectangular distribution. The wakefield generates an energy change, different for each time-slice along the bunch, potentially shifting the slice out of resonance, and reducing the total FEL power. The low charge configuration does not generate such high current spikes, and produces smaller wakefields in the undulator allowing more of the bunch to 


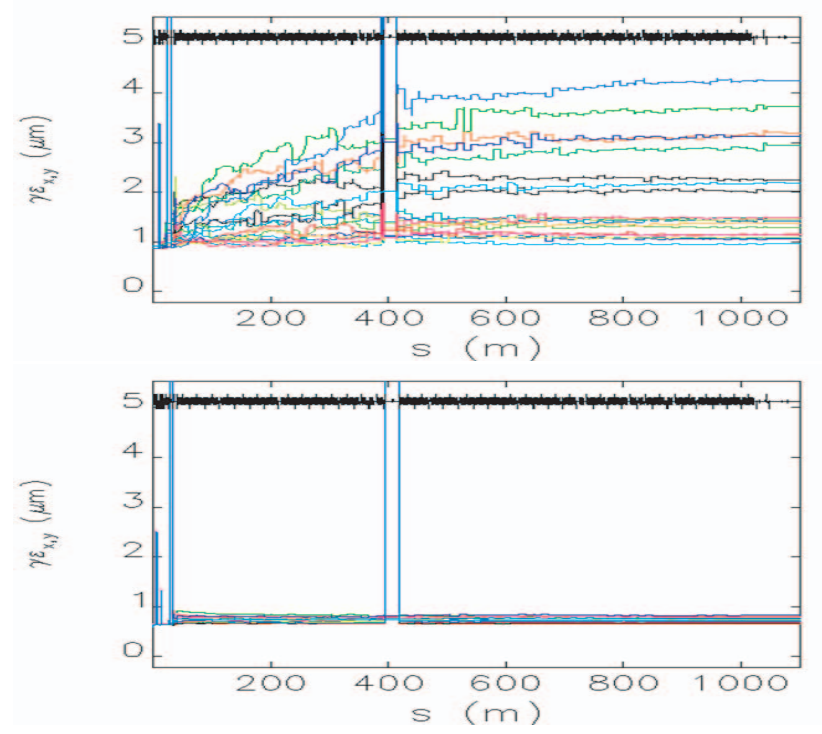

Figure 4: Normalized emittance along linac with a 1-nC (top) and 0.2-nC (bottom). The linac quadrupoles, BPMs, and RF structures are all misaligned by $250 \mu \mathrm{m}$ rms and the trajectory is corrected for 10 different seeds.

reach saturation. The Fig. 5 plots are for a cylindrical copper pipe with $2.5-\mathrm{mm}$ radius and $130-\mathrm{m}$ length.

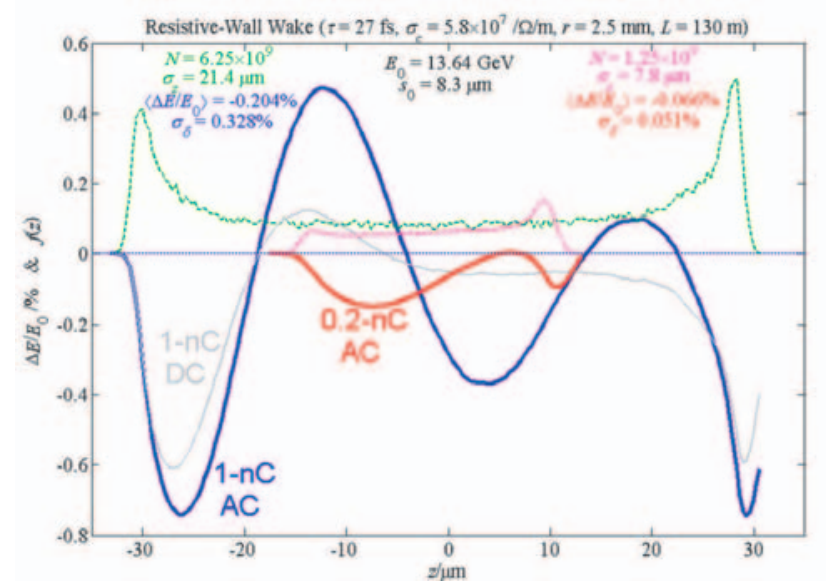

Figure 5: Resistive-wall wakefield for both the 1-nC (blue) and 0.2-nC (red) cases. Both longitudinal distributions are also shown (dashed lines). Bunch head at $z<0$.

\section{FEL SIMULATIONS}

The particle tracking in elegant is used as input to the Genesis and Ginger FEL codes to evaluate the FEL gain and $\mathrm{x}$-ray power [11]. The resistive wakefield is included in these calculations, as is a variable slow field taper along the undulator to allow compensation for beam energy loss due to wakes and spontaneous radiation. The time-sliced transverse emittance predicted by Parmela for the 0.2-nC case at a 30 -A peak current is $\gamma \epsilon_{x, y} \approx 0.6 \mu \mathrm{m}$, including a large thermal emittance of $1 \mu \mathrm{m}$ per millimeter of cathode laser-illumination radius $\left(\Delta \gamma \epsilon_{t h} \approx 0.42 \mu \mathrm{m}\right)$. In order to provide a more conservative picture, we arbitrarily scaled this time-slice emittance up to the $0.8-\mu \mathrm{m}$ level originally anticipated, before tracking in elegant.

Figure 6 shows the FEL $x$-ray power at $1.5 \AA$ along the pulse length, calculated using the distributions from the $e l$ egant tracking. (The plot was artificially smoothed with 1-fs resolution). The optimal field taper (the equivalent of $300 \mathrm{kV} / \mathrm{m}$ ) produces about $20 \mathrm{GW}$ over the whole pulse length, whereas no field taper produces almost 10-times less power. The optimal taper is about twice the level of the average resisitive-wake loss, and is in agreement with Ref. [12].

Since the large wakefield in the 1-nC case only allows saturation for a fraction of the bunch, the $\mathrm{x}$-ray pulse energy for the 1-nC and $0.2-n C$ cases are actually quite similar, within about a factor of two, with at least $10^{12}$ photons per pulse. Finally, the low-charge case also produces a shorter x-ray pulse, about 70-fs FWHM as opposed to 200-fs FWHM in the 1-nC case.

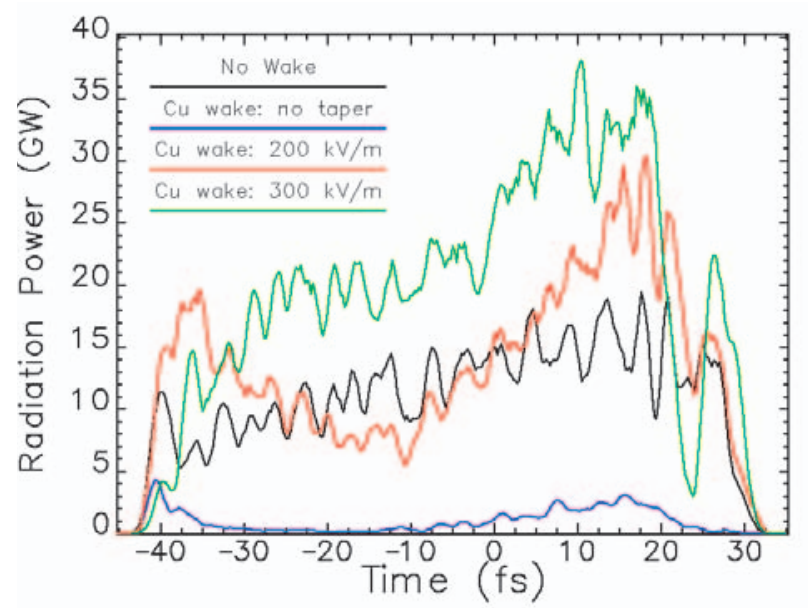

Figure 6: Ginger FEL calculations of the 0.2-nC case, including resistive wakefield losses along the undulator and various magnetic field tapers to optimize the power.

\section{REFERENCES}

[1] LCLS CDR, SLAC Report No. SLAC-R-593, 2002.

[2] TESLA TDR, DESY Report No. DESY-2001-011, 2001.

[3] M. Xie, PAC'95, 1995, p. 183.

[4] C. Limborg-Deprey et al., these PAC'05 proceedings.

[5] K. Bane et al., PAC'93, 1993, p. 596.

[6] J. Billen, LANL Report LA-UR-96-1835 (1996).

[7] K. Bane and P. Emma, these PAC'05 proceedings.

[8] M. Borland, ANL/APS LS-287, Sep. 2000.

[9] Z. Huang et al., PRST-AB, 7, 074401 (2004).

[10] K. Bane and G. Stupakov, these PAC'05 proceedings.

[11] S. Reiche et al., these PAC'05 proceedings.

[12] Z. Huang and G. Stupakov, these PAC'05 proceedings. 